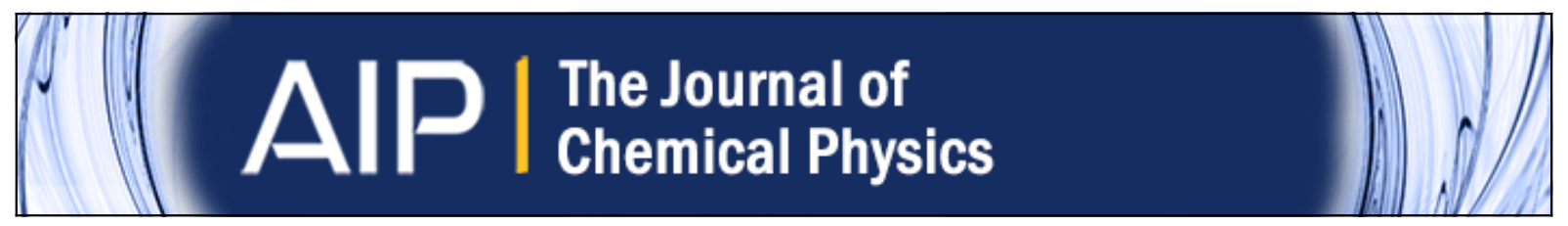

\title{
Nucleation of wetting films on cylindrical and spherical substrates: A numerical study by the string method
}

Chunyin Qiu and Tiezheng Qian

Citation: The Journal of Chemical Physics 131, 124708 (2009); doi: 10.1063/1.3239462

View online: http://dx.doi.org/10.1063/1.3239462

View Table of Contents: http://scitation.aip.org/content/aip/journal/jcp/131/12?ver=pdfcov

Published by the AIP Publishing

\section{Articles you may be interested in}

Numerical study of the effects of surface topography and chemistry on the wetting transition using the string method

J. Chem. Phys. 141, 244705 (2014); 10.1063/1.4904947

Theoretical description of the adsorption and the wetting behavior of alkanes on water

J. Chem. Phys. 125, 084718 (2006); 10.1063/1.2338806

Wetting transitions of ionic solutions

J. Chem. Phys. 121, 3613 (2004); 10.1063/1.1772360

Heterogeneous nucleation and wetting of water thin films on a metal surface: A study by optical second harmonic generation

J. Chem. Phys. 118, 5106 (2003); 10.1063/1.1553793

Wetting on a geometrically structured substrate

J. Chem. Phys. 115, 6200 (2001); 10.1063/1.1399037

How can you REACH $100 \%$

of researchers at the Top 100

Physical Sciences Universities?

With The Journal of Chemical Physics.

AIP $\left.\right|_{\text {Che Journal of }} ^{\text {Theal Physics }}$

THERE'S POWER IN NUMBERS. Reach the world with AIP Publishing.

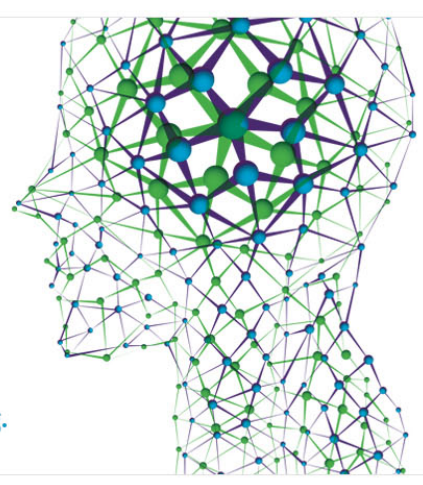




\title{
Nucleation of wetting films on cylindrical and spherical substrates: A numerical study by the string method
}

\author{
Chunyin Qiu ${ }^{1}$ and Tiezheng Qian ${ }^{2, a)}$ \\ ${ }^{1}$ Program in Nano Science and Technology, Hong Kong University of Science and Technology, \\ Clear Water Bay, Kowloon, Hong Kong \\ ${ }^{2}$ Department of Mathematics and Joint KAUST-HKUST Micro/Nano-Fluidics Laboratory, \\ Hong Kong University of Science and Technology, Clear Water Bay, Kowloon, Hong Kong
}

(Received 6 July 2009; accepted 9 September 2009; published online 25 September 2009)

\begin{abstract}
Using the mean-field diffuse-interface model for liquid-vapor system and employing the numerical string method, we study the critical nuclei involved in the prewetting transitions on curved substrates. We first introduce three distinct kinds of critical nuclei, namely, the disklike, bandlike, and layerlike ones, which respectively correspond to three possible growth modes of wetting films. We show the disklike growth mode to be the only mode for infinite planar substrates. We then turn to cylindrical and spherical substrates, the two simplest but most important geometries in the real world. We focus on the critical nuclei of finite size, through which the wetting films may be formed with finite thermodynamic probabilities. It is shown that the disklike growth mode is always the most probable for wetting film nucleation and growth as long as a disklike critical nucleus exists. It is also shown that on a cylindrical substrate, the disklike critical nucleus can no longer exist if the substrate radius is smaller than some critical value, comparable to the radius of the disklike critical nucleus on planar substrate. We find that on a cylindrical substrate whose radius is below the critical value, the nucleation and growth of a wetting film can only occur through the bandlike critical nucleus. It is worth emphasizing that the results concerning the bandlike and layerlike growth modes can only be obtained from the diffuse-interface model, beyond the macroscopic description based on the line and surface tensions. (C) 2009 American Institute of Physics. [doi:10.1063/1.3239462]
\end{abstract}

\section{INTRODUCTION}

In contact with a solid surface, a liquid-vapor interface may exhibit either a finite equilibrium contact angle (partial wetting) or a zero contact angle (complete wetting). A particular temperature, called the wetting transition temperature, may exist that separates one regime from the other. ${ }^{1-3}$ The wetting transitions in fluid systems in contact with solid substrates have attracted much attention for decades due to both fundamental theoretical interests and extensive industrial applications (e.g., lubrication and adsorption). For first-order wetting transitions at two-phase coexistence, it has been predicted that at temperatures/compositions, where the wetting phase is no longer stable in bulk, there exists a first-order transition associated with a finite jump in adsorption, between a "weak" phase of thin film and a "strong" phase of thick film. ${ }^{1-23}$ The line of this first-order surface transition is known as the prewetting line, which meets the coexistence axis at the first-order wetting transition. As the fluid density is only slightly enhanced near the substrate, the thin film phase can be viewed as a perturbed vapor phase. To avoid confusion, here we refer to the thin film phase as the vapor phase and retain the term of film phase for the thick film phase. For the static properties of wetting and prewetting transitions, most of the existing theoretical ${ }^{1-13}$ and experimental ${ }^{14-17}$ investigations have been focused on

\footnotetext{
${ }^{a)}$ Author to whom correspondence should be addressed. Electronic mail: maqian@ust.hk.
}

systems with the simplest geometry-infinite planar surface-and considerably less attention has been paid to systems of other surface geometries, e.g., finite and/or curved surfaces that deviate from the "ideal" case of planar substrate but are familiar in the real world. ${ }^{18-23}$ For a fluid system in contact with a uniformly curved substrate, such as a cylinder or a sphere, the wetting transition, which would occur at an infinite planar surface, has been shown to be suppressed by the nonzero curvature and the finite size of the substrate, whereas the prewetting transition remains. ${ }^{19,20}$

In a first-order prewetting transition, the film phase is formed from the vapor phase through a thermally activated critical nucleus, statistically a rare event that involves an energy barrier crossing. In the description of the nucleation process, the critical nucleus plays the central role. Those measurable physical quantities, e.g., the rate of nucleation which is the frequency of the appearance of critical nuclei per unit volume/area of the system under consideration, are completely determined by the properties of the critical nucleus. In the classical nucleation theory, the film phase nucleates from a liquid disk that is of some critical size, created by large thermal fluctuations. Once such a critical nucleus is created, from it the wetting film would grow spontaneously. This classical picture is derived in a macroscopic description ${ }^{14,16,17}$ based on the concepts of surface tension and line tension, with the critical size of the liquid disk determined by a competition between the additional positive line energy and negative surface energy. The macroscopic approach is particularly suitable for systems close to the 
binodal point (i.e., the coexistence of the vapor and film phases), with the critical nuclei being macroscopically large. It fails, however, when the spinodal point (i.e., the boundary of metastability) is approached and as a consequence the critical nucleus consists of only a small number of molecules, making the definitions of surface and line tensions questionable. Moreover, because of the oversimplified description in the macroscopic approach (e.g., at an infinite planar surface the liquid disk is characterized by its radius only), some physically possible transition pathways may be lost, although they can be especially important to systems of finite size, as to be shown in this study. In order to overcome these limitations and/or shortcomings, people have turned to the continuum description of fluid systems by free energy functional, from either the density-functional theory ${ }^{4-7,22}$ or the (Landau) mean-field theory. ${ }^{1-3,12,13,18-20,23}$ In this description, the critical nucleus corresponds to a saddle point of the grand potential functional. Some earlier mean-field studies have been focused on systems on infinite planar substrates. ${ }^{8-12}$

In the present study, we adopt the mean-field theory to investigate the nucleation processes for wetting films on spherical and cylindrical substrates, which are the most representative examples of curved and finite surfaces. To the best of our knowledge, this is the first mean-field study for the critical nuclei involved in prewetting transitions on curved substrates. In this approach, the critical nucleus corresponds to a saddle point of the grand potential functional, which is an unstable, stationary solution of the EulerLagrange equation, thus hard to solve numerically. An efficient numerical method for locating the saddle points in grand canonical ensemble is indispensable in reaching a quantitatively accurate continuum description of critical nuclei. Recently, the string method ${ }^{24,25}$ has been presented for the numerical evaluation of thermally activated rare events. By evolving strings, defined as smooth curves with intrinsic parameterization (such as arc length) in configuration space, toward the minimal energy path (MEP), the string method locates the most probable transition pathway connecting two metastable/stable states in configuration space. The saddle point corresponds to the maximum energy (here the maximum grand potential) along the MEP. The string method has been shown to be an efficient numerical tool for evaluating the thermally activated phase slips in low-dimensional superconductors. ${ }^{26,27}$ The applicability and power of string method in locating the saddle points in fluid systems have already been demonstrated in the study of capillary condensation for fluids confined in slit pores. ${ }^{28}$ In this work, we apply the string method to study the critical nuclei in prewetting transition. We will locate different MEPs that correspond to different growth modes of wetting films on substrates of different geometries.

The paper is organized as follows. In Sec. II, we first outline the continuum model for the mean-field approach. We then introduce three distinct kinds of critical nuclei, namely, the disklike, layerlike, and bandlike ones, which correspond to three different growth modes of wetting films. The relevance of these growth modes to systems with cylindrical and spherical surfaces is discussed qualitatively. We present the numerical results in Sec. III. By using the string method, we calculate the saddle points of grand potential functional and the corresponding grand potential barriers. We focus on the critical nuclei of finite size, through which the nucleation may occur with finite probabilities. Our calculations show the disklike growth mode (DGM) to be the most probable one for forming the wetting film as long as it exists (on substrates of sufficiently large extension). Our calculations also show that in the case of cylindrical substrate, the disklike critical nucleus may cease to exist, depending on the system size. Once it disappears, the nucleation can only be realized through the bandlike growth mode (BGM). It is important to note that for the BGM, there is no simple macroscopic description (in terms of surface and line tensions) because of the very limited spatial extension of the critical nucleus. In this case the nucleation process has to be described by the spatial distribution of fluid density in a meanfield or density-functional approach. Following the case of cylindrical substrate, the nucleation of wetting film on spherical substrate is also presented, where the three distinct growth modes are compared. The paper is concluded in Sec. IV.

\section{MEAN-FIELD THEORY}

\section{A. Continuum model}

The mean-field theory has been extensively used to investigate the wetting phenomena for fluid systems. ${ }^{1-3,12,13,18-20,23}$ We consider a fluid undersaturated with respect to the bulk liquid phase. With $\phi(\mathbf{r})$ representing the local relative density of fluid, the grand potential for an inhomogeneous fluid is a functional of the density profile,

$$
\Omega_{f}[\phi(\mathbf{r})]=\int_{V}\left[f(\phi)-\mu_{d} \phi+\frac{\kappa}{2}(\nabla \phi)^{2}\right] d \mathbf{r},
$$

where $f(\phi)=-\alpha \phi^{2} / 2+\beta \phi^{4} / 4$ is the grand potential density for a fluid of density $\phi$ at the liquid-vapor bulk coexistence $\mu_{c}, \mu_{d}=\mu-\mu_{c}$ is the chemical potential difference measured relative to $\mu_{c}$ (with the undersaturated vapor phase corresponding to a negative value of $\left.\mu_{d}\right)$, and $\kappa(\nabla \phi)^{2} / 2$ measures the excess free energy density associated with the density inhomogeneity, with $\alpha, \beta$, and $\kappa$ being positive phenomenological parameters. The present continuum model is also called the diffuse-interface model in which interfaces have a nonzero thickness, i.e., they are "diffused," so that all quantities vary continuously. In the grand potential expressed in Eq. (1), the density function $f(\phi)$ of a symmetric double-well structure determines the two equilibrium values $\phi_{ \pm}$of the relative density $\phi$ at bulk coexistence, with $\phi_{+}=(\alpha / \bar{\beta})^{1 / 2}$ and $\phi_{-}=-(\alpha / \beta)^{1 / 2}$ corresponding to the liquid and vapor phases, respectively. As the system is biased by the chemical potential undersaturation $\mu_{d}<0$, the liquid phase is metastable or even unstable, depending on the value of $\left|\mu_{d}\right|$. The squaregradient approximation for $\kappa(\nabla \phi)^{2} / 2$ is based on the assumption that the characteristic distance over which $\phi(\mathbf{r})$ varies is much larger than molecular length scale, as usually assumed in the mean-field description.

For a fluid in contact with a solid substrate, the additional free energy due to the substrate-fluid interactions, 


$$
\Omega_{s f}=\int_{S} \Phi\left(\phi_{s}\right) d \mathbf{a}
$$

needs to be introduced. Here the substrate-fluid interactions are assumed to be short ranged, and hence the surface free energy per unit area of the substrate, denoted by $\Phi$, only depends on the fluid density at solid surface $\phi_{s}$ in the form of $\Phi\left(\phi_{s}\right)=-\gamma \phi_{s}-g \phi_{s}^{2} / 2$, where $\gamma$ is a measure of the surface field and $g$ accounts for the possible enhancement of molecular interactions at the substrate. ${ }^{3}$ For simplicity, in this paper we take the $\gamma$ term only and assume $g=0$. Surface free energy functions can be written down in some more general forms that include longer-range substrate-fluid (van der Waals) interactions. However they are not considered here. Equilibrium properties of this system have been extensively investigated, ${ }^{1-7,18-23}$ especially for the case of infinite planar substrate. ${ }^{1-7}$ Compared to the pure fluid system, the phase diagram in the presence of a substrate is much richer because of the competition between the bulk and surface free energies. Besides the ordinary vapor and liquid phases, there may exist a new phase that involves a liquid film at solid surface. That is, for small undersaturation $\left(\mu_{d}<0\right)$ but strong attractive surface field $\left[\gamma>0\right.$ in $\Phi\left(\phi_{s}\right)$, with complete wetting at the liquid-vapor bulk coexistence], the stable phase is the film phase, characterized by an intruding liquid film between the solid surface and the bulk vapor. The surface transition between the vapor phase and the film phase is referred to as the prewetting transition. It is instructive to note that the surface field $\gamma$ plays a role similar to the relative chemical potential $\mu_{d}$ although the former is localized at the solid surface. Physically, the prewetting transition results from a competition between the attractive surface potential (with $\gamma>0$ ) which prefers the high-density liquid phase and the chemical potential undersaturation (with $\mu_{d}<0$ ) which prefers the low-density vapor phase. That the surface field is localized is reflected in the finite thickness of the wetting film.

For computational purpose, we use the dimensionless form

$$
\bar{\Omega}=\int_{\bar{V}}\left[(\bar{\nabla} \bar{\phi})^{2} / 2-\bar{\phi}^{2} / 2+\bar{\phi}^{4} / 4-\bar{\mu}_{d} \bar{\phi}\right] d \overline{\mathbf{r}}-\int_{\bar{S}} \bar{\gamma} \bar{\phi}_{S} d \overline{\mathbf{a}}
$$

for the total grand potential $\Omega=\Omega_{f}+\Omega_{s f}$. Here the over bars denote those dimensionless quantities obtained with the lengths scaled by the correlation length $\xi=(\kappa / \alpha)^{1 / 2}$, which serves as the length scale for the liquid-vapor interfacial thickness, the grand potential $\Omega$ by $\alpha^{2} \xi^{3} / \beta$, the fluid density $\phi$ by $\phi_{+}=(\alpha / \beta)^{1 / 2}$, the chemical potential difference $\mu_{d}$ by $\alpha(\alpha / \beta)^{1 / 2}$, and the attraction strength of surface potential $\gamma$ by $\alpha(\kappa / \beta)^{1 / 2}$. Throughout the remainder of this paper, we will use the dimensionless quantities with the overbars all dropped. We want to point out that in the dimensionless description, all the temperature effects have already been adsorbed into the various units that are used in defining the dimensionless quantities.

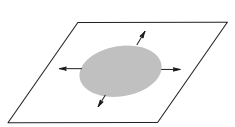

(a)

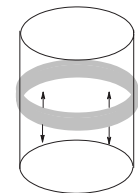

(b)

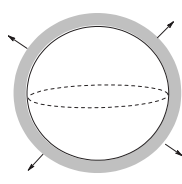

(c)
FIG. 1. A schematic illustration for different growth modes on different substrates. (a) The DGM on a large planar substrate. (b) The BGM on a thin cylindrical substrate. (c) The LGM on a small spherical substrate.

\section{B. Metastable and stable phases and saddle points}

Extremization of the total grand potential with respect to the density field $\phi(\mathbf{r})$ leads to the Euler-Lagrange equation

$$
-\frac{\delta \Omega[\phi(\mathbf{r})]}{\delta \phi(\mathbf{r})}=\nabla^{2} \phi+\phi-\phi^{3}+\mu_{d}=0
$$

in the bulk and the boundary condition $\left.\partial_{n} \phi\right|_{s}=\gamma$, where $\left.\partial_{n} \phi\right|_{s}$ denotes the derivative along the outward surface normal at the solid surface (with $n$ pointing into the solid). Due to the attractive surface potential, the fluid density exhibits shortrange variations near the solid surface in stable and metastable (if exists) phases. Numerically, the stable and metastable density profiles can be calculated by the method of steepest decent provided an appropriate initial profile is given. For the convenience of presentation, we let $\Omega_{V}$ denote the grand potential minimum for the vapor phase and $\Omega_{F}$ denote that for the film phase. The prewetting line is determined by a comparison between these two minima. In the study of the nucleation of wetting films, we assume the vapor phase to be metastable (i.e., $\Omega_{F}<\Omega_{V}$ ). It is from this metastable initial state that the stable film phase is to be reached through a nucleation process, characterized by a critical nucleus.

Being a solution of the Euler-Lagrange equation, the saddle point of the total grand potential gives the critical nucleus which physically corresponds to the most probable thermally activated fluctuation that can carry the system from the metastable vapor phase to the stable film phase. Below we propose three different growth modes for the wetting films based on the consideration of translational symmetry and its breaking (see Fig. 1 for a schematic illustration). We start from the ideal case of infinite planar substrate and then move to the cylindrical and spherical substrates. We will show that the existence one or two finite dimensions of a substrate would allow the occurrence of those growth modes that are prohibited at the infinite planar substrates.

In terms of translational symmetry and its breaking, there may exist three distinct kinds of saddle points in the functional space of the density field $\phi(\mathbf{r})$. Saddle points of each kind are characterized by the translational symmetry along the surface or its breaking along one or two directions, and correspond to a particular way for the nucleation and subsequent growth of wetting films. Saddle points of the first kind exhibit the translational symmetry along the surface, with $\phi(\mathbf{r})$ only varying along the (local) surface normal of 
the substrate. We call these saddle points the layerlike solutions because as (grand potential) barriers, they would be crossed in a layer-by-layer growth of wetting film. Saddle points of the second kind exhibit translational symmetry breaking along one direction parallel to the surface. Consequently, these saddle points are characterized by density profiles that look like a liquid band, with the translational symmetry preserved in one direction (along the band) but broken in the other direction (across the band). According to this picture, we call these saddle points the bandlike solutions because they would be involved in the growth of those wetting films that spread bilaterally in the transversal direction across the liquid band. Since the formation of critical nucleus is a thermally activated rare event, the rate of nucleation $\Gamma$ is proportional to the exponential factor $\exp \left(-\Delta \Omega / k_{B} T\right)$, i.e., $\Gamma=v \exp \left(-\Delta \Omega / k_{B} T\right)$, in which $v$ is the so-called prefactor and $\Delta \Omega$ is the grand potential barrier. For a saddle point of the first or the second kind, it is obvious that the grand potential barrier for nucleation, i.e., $\Delta \Omega_{L}=\Omega_{S}^{L}-\Omega_{V}$ for the layerlike growth mode (LGM) or $\Delta \Omega_{B}=\Omega_{S}^{B}-\Omega_{V}$ for the BGM, is proportional to the area or the length of the substrate, which is infinite in the case of infinite planar substrate. Here $\Omega_{S}^{L}$ and $\Omega_{S}^{B}$ denote the grand potentials of the layerlike and bandlike saddle points, respectively. The infinite $\Delta \Omega_{L}$ and $\Delta \Omega_{B}$ mean that on an infinite planar substrate, a prewetting transition will never occur without breaking the translational symmetry along both directions parallel to the surface. We are therefore led to saddle points of the third kind, characterized by density profiles possessing a structure that breaks the translational symmetry along two directions. In the case of infinite planar substrate, the localized structure exhibits cylindrical symmetry, and the corresponding critical nuclei are circular liquid disks. They would be involved in the growth of those wetting films that spread along the radial direction of the liquid disk [see Fig. 1(a)]. The finite size of the critical nucleus results in a finite grand potential barrier $\Delta \Omega_{D}=\Omega_{S}^{D}$ $-\Omega_{V}$, with $\Omega_{S}^{D}$ denoting the grand potential of the disklike saddle points, and hence a finite probability. We call these saddle points the disklike solutions (although cylindrical symmetry may not necessarily be always present for substrates other than the infinite planar ones). We note that saddle points of the third kind have been extensively discussed in literatures, based on the sharp-interface model ${ }^{8-11}$ and the mean-field diffuse-interface model. ${ }^{12,13}$

For curved substrates that are finite along one or two directions parallel to the surface, there can be more growth modes available to wetting films. In particular, for cylindrical substrates, the bandlike critical nuclei may be of finite size with the liquid band forming a circle around the cylinder [see Fig. 1(b)], and hence they may be created with finite probabilities through large thermal fluctuations. Moreover, for spherical substrates, either the bandlike or the layerlike critical nuclei become finite [see Fig. 1(c) for the layerlike nuclei]. Being thermally activated rare events, different growth modes compete in terms of the grand potential barrier. Intuitively, for a cylindrical/spherical substrate with the cylindrical/spherical radius being much larger than the radius of the disklike critical nucleus (on infinite planar substrate), the curvature effect can be neglected and the DGM domi- nates. For curved substrates of short extension(s) along one or two directions, however, the curvature and finite size effects may greatly change the growth behavior of wetting films, and the competition among those physically feasible growth modes has to be quantitatively evaluated. Numerical results in this regard will be presented in Sec. III. It is worth emphasizing that the limited spatial extension(s) of a curved substrate may invalidate the concepts of surface tension and line tension used in the macroscopic description. This necessitates the mean-field diffuse-interface description here.

We want to point out that in terms of numerical computation, it is not an easy task to locate the saddle points which are actually unstable stationary points satisfying the EulerLagrange equation. ${ }^{4}$ This is true even in the case of infinite planar substrate. ${ }^{12}$ In Sec. III, the various critical nuclei in prewetting transitions will be numerically located by employing the string method, ${ }^{24-27}$ which has already been applied to fluid systems for the study of capillary condensation in slit pores. ${ }^{28}$ Different transition pathways (i.e., MEPs) will be presented, showing different growth modes of wetting films on substrates of different geometries. An outline for the string method and its application can be found in Refs. 26 and 28 .

\section{NUMERICAL RESULTS}

We consider an unsaturated fluid in contact with a substrate. Besides the substrate geometry, the system is controlled by two dimensionless parameters: $\mu_{d}$ for the chemical potential difference relative to the liquid-vapor bulk coexistence and $\gamma$ for the strength of attractive surface potential. Without losing generality, in the present numerical study we fix $\mu_{d}=-0.04$ and set $\gamma$ to be greater than some critical value $\gamma_{o}$ that corresponds to the binodal of a prewetting transition (at which $\Omega_{F}=\Omega_{V}$ ), e.g., $\gamma_{o} \cong 0.615$ for the case of infinite planar substrate. ${ }^{28}$ By $\gamma>\gamma_{o}$ the film phase is stabilized with $\Omega_{F}<\Omega_{V}$. Given the metastable vapor phase as the initial state, the system is expected to reach the stable film phase through the nucleation of a wetting film. With the numerical details already presented elsewhere, ${ }^{28}$ the string method is implemented through a finite difference scheme. The real space is discretized by a mesh of $M$ points. A configuration $\phi(\mathbf{r})$ is represented by a column vector of $M$ entries and the grand potential $\Omega[\phi(\mathbf{r})]$ becomes a multivariable function. Each discretized by $N$ points in the configuration space (now a $M$ dimensional vector space), strings (i.e., smooth curves) are parametrized by the arc length $s$. A reparametrization is applied once in a while during the evolution of a discretized string toward the MEP.

\section{A. Nucleation on infinite planar substrates}

We first consider the fluid on an infinite planar substrate. As discussed in Sec. II B, a wetting film can be formed with finite probability only by crossing a saddle point characterized by a liquid disk. To numerically present the disklike saddle point, we make use of the cylindrical symmetry exhibited in the nucleation of wetting film and adopt a twodimensional (2D) cylindrical coordinate system $(r, z)$, with $z$ being the normal distance to the substrate and $r$ the radial 

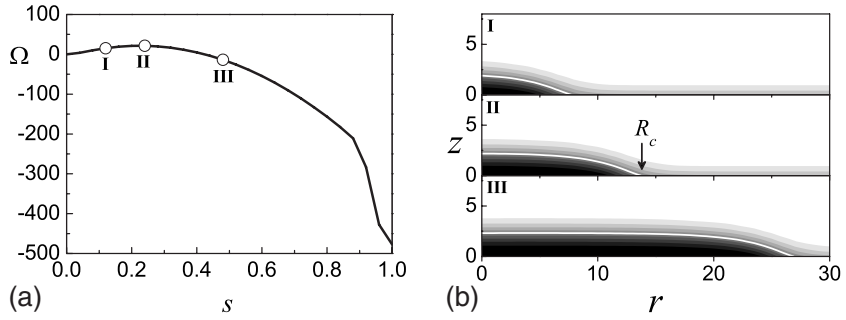

FIG. 2. (a) The total grand potential plotted as a function of the arc length $s$ in the configuration space, evaluated for $\mu_{d}=-0.04$ and $\gamma=0.65$. Here the value of the total grand potential in the initial vapor phase $\Omega_{V}$ has been subtracted to let the curve start from zero. (b) The density profile $\phi(r, z)$ plotted for a sequence of states labeled along the curve in (a), with the gray scale varying from black for liquid phase $\left(\phi_{+}=1\right)$ to white for vapor phase $\left(\phi_{-}=-1\right)$. In particular, the level curve $\phi=0$ is marked by the white line.

distance measured from some fixed origin. We take $\gamma=0.65$ to stabilize the film phase with $\Omega_{F}<\Omega_{V}$. Figure 2(a) shows the variation of total grand potential $\Omega$ along the MEP that starts from the vapor phase in the configuration space. The grand potential barrier associated with the transition from vapor phase to film phase is evaluated to be $\Delta \Omega_{D}=\Omega_{D}^{S}$ $-\Omega_{V} \cong 21.44$. Displayed in Fig. 2(b) is a sequence of three states along the MEP, showing clearly the formation of a disklike critical nucleus and the subsequent growth of wetting film from it. In particular, state II corresponds to the critical nucleus, i.e., the saddle point, which is of the highest grand potential along the MEP. The nominal location of liquid-vapor interface is determined by the level curve $\phi=0$ [marked by the white line in Fig. 2(b)], from which the radius of the critical nucleus is estimated to be $R_{c} \cong 13.5$.

The disklike critical nucleus can be easily described using the concepts of surface tension and line tension. In this macroscopic description, the excess free energy of a liquid disk formed through thermal fluctuations is given by $E=l \widetilde{\tau}-A \widetilde{S},{ }^{14,16,17}$ where $l$ and $A$ are the circumference and surface area of the liquid disk, respectively, $\widetilde{\tau}$ is the line tension, and $\tilde{S}$ is the positive spreading coefficient. On the planar substrate, the liquid disk is circular (to minimize the circumference for a given area), characterized by a radius $R$. Maximizing $E=l \widetilde{\tau}-A \widetilde{S}$ with respect to the disk radius $R$ determines the critical radius $R_{c}=\widetilde{\tau} / \widetilde{S}$, which is a $2 \mathrm{D}$ analog of the Laplace equation. The maximum excess free energy at $R_{c}$ is $E_{c}=\pi \widetilde{\tau} / \tilde{S}$. Once the critical nucleus is formed through thermal fluctuations, the wetting film spreads spontaneously over the substrate. Note that in this macroscopic description, the size of the liquid disk must be macroscopically large with $R \gg 1$ (scaled by the correlation length $\xi$ ) to ensure that the surface and line tensions are well defined material constants. While the macroscopic parameters $\tilde{S}$ and $\tilde{\tau}$ can be obtained from the mean-field calculations, here we will not present numerical results in this regard.

A similar macroscopic description can be applied to the nucleation of wetting films on curved substrates, provided the curvature radius is much larger than the critical radius $R_{c} \cong 13.5$. On these weakly curved substrates, the DGM remains to be the most probable growth mode, and the finite size effect shows up only when a spreading liquid disk/film approaches the finite substrate extension(s) in one or more directions in the later stage of spontaneous film growth. On the other hand, the saddle point and hence the grand potential barrier would be considerably changed if the substrate has finite extension(s) in one or more directions that are comparable to or even smaller than the critical radius $R_{c}$. In fact, as discussed in Sec. II B, growth modes other than the DGM, e.g., the LGM and BGM, may arise. Below we focus on the finite size effects of cylindrical and spherical substrates on the nucleation of wetting films.

\section{B. Nucleation on cylindrical substrates}

It has been well known that the curved geometry acts as an effective bulk field which drives the system away from two-phase coexistence, and thus suppresses complete wetting. This may be understood by observing that a thicker wetting film bears a liquid-vapor interface of larger area. Nevertheless, the prewetting transition still remains. ${ }^{19,20}$ Here we focus on how the finite size of a curved substrate would affect the nucleation pathways in prewetting transition. For the cylindrical substrate of infinite length, the LGM of wetting film involves a saddle point that is infinitely long in real space. This leads us to consider the DGM and BGM only. The curvature effect can be easily incorporated. However, in order to make a more direct comparison with the case of infinite planar substrate, here we ignore the substrate curvature in calculations. That is, the cylindrical substrate of radius $R_{\text {cyl }}$ is geometrically viewed as a $2 \mathrm{D}$ planar strip of width $C=2 \pi R_{\text {cyl }}$, supplemented with the periodic boundary condition in the transversal direction (i.e., the circumferential direction of cylinder). It is noted that the approximation is valid if $h \ll R_{\text {cyl }}$, where $h$ is the thickness of wetting film. It is natural to adopt the rectangular coordinate system $(x, y, z)$, with $x$ and $y$ measured along the length and width directions of the strip (i.e., the longitudinal and circumferential directions of the cylinder), and $z$ along the direction normal to the strip. Using $\mu_{d}=-0.04$ and $\gamma=0.65$ again, we consider a cylindrical substrate of circumference $C=30$, a bit larger than $2 R_{c} \cong 27$, the diameter of the disklike critical nucleus on the infinite planar substrate [see Fig. 2(b)]. [Here the condition $h \ll R_{\text {cyl }}$ for strip approximation is not well satisfied by the film thickness $h \cong R_{\text {cyl }} / 3$. However this will not qualitatively change the results presented below on how the finite circumference of a cylindrical substrate would affect the nucleation pathways.] Using initial strings particularly prepared, we have numerically obtained two distinct MEPs corresponding to the DGM and BGM, respectively. From each MEP a saddle point can be located and the corresponding grand potential barrier evaluated [see Fig. 3(a)]. The barrier for the DGM, $\Delta \Omega_{D}=\Omega_{S}^{D}-\Omega_{V}$, is evaluated to be $\cong 21.39$, which is a bit smaller than that obtained for the infinite planar substrate

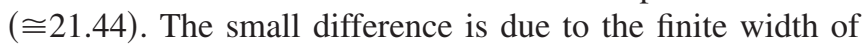
the system by which the disklike critical nucleus is deformed, as to be shown below. The barrier for the BGM, $\Delta \Omega_{B}=\Omega_{S}^{B}-\Omega_{V}$, is evaluated to be $\cong 23.63$, which is larger than $\Delta \Omega_{D}$. Therefore, at $C=30$ the nucleation of a wetting film is still more probable to occur through the DGM.

Figures 3(b) and 3(c) show how the nucleation of a wetting film proceeds on a strip substrate with the periodic 

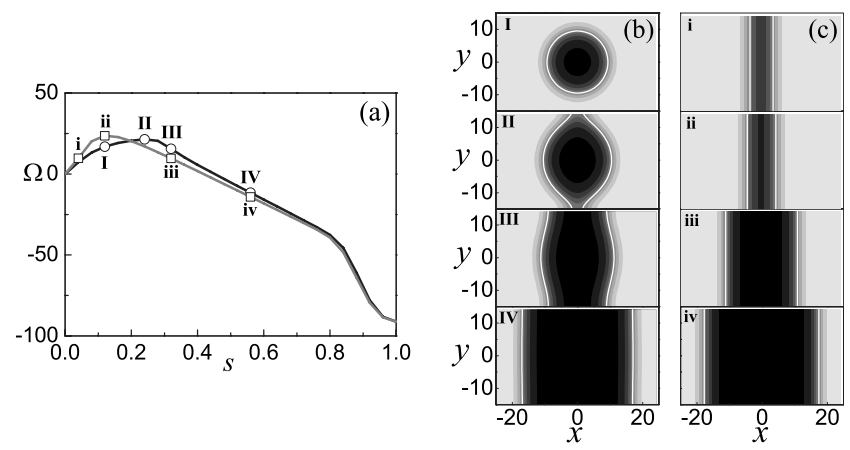

FIG. 3. (a) The total grand potential plotted as a function of the arc length $s$ in the configuration space, evaluated for $\mu_{d}=-0.04, \gamma=0.65$, and $2 \pi R_{\text {cyl }}=30$. The black line represents the MEP passing through the disklike saddle point, and the gray line represents the MEP through the bandlike saddle point. For each curve, the value of the total grand potential in the initial vapor phase $\Omega_{V}$ has been subtracted. [(b) and (c)] The density distribution taken at the substrate surface $(z=0)$, plotted for a sequence of states labeled in (a), with the gray scale varying from black for liquid phase $\left(\phi_{+}=1\right)$ to white for vapor phase $\left(\phi_{-}=-1\right)$. In particular, the level curve $\phi=0$ is marked by the white line.

boundary condition in the transversal direction (approximately a cylindrical substrate). Taken at the substrate surface $z=0$, the density profiles are presented for a sequence of states labeled along the MEP, with states I-IV for the DGM and states i-iv for the BGM, respectively. Figure 3(b) shows that in the case of DGM, the nucleation starts from a small (almost perfect) circular liquid disk (state I). Once the two boundaries of the growing disk in the $y$ direction become in contact with each other due to the periodical boundary condition, the circular shape is deformed (state II), followed by a gradual reduction of the curvature (state III). In a macroscopic point of view, this curvature reduction is to decrease the positive free energy due to the line tension. Later on, the wetting film spreads along the $\pm x$ directions with two fronts that are almost straight in the $y$ direction (state IV). We want to point out that due to the finite extension along $y$, the disklike critical nucleus, state II, is no longer perfectly circular, and this shape distortion is beyond the macroscopic description in which a circular critical nucleus is always predicted. This necessitates the present continuum model in which the liquid-vapor interface is diffuse. Figure 3(c) shows that in the case of BGM, the nucleation starts from a liquid band with $\phi(\mathbf{r})$ uniformly distributed in the $y$ direction (state i). This liquid band then widens bilaterally along the $\pm x$ directions (states ii-iv). In particular, state ii corresponds to the critical nucleus. It is worth emphasizing that the BGM displayed in Fig. 3(c) can, by no means, be explained in the macroscopic description. This can be understood as follows. With the excess free energy written as $E=2 C \widetilde{\tau}-C w_{B} \widetilde{S}$, where $w_{B}$ is the $x$ extension of the liquid band, it is noted that the first term due to the line tension $\widetilde{\tau}$ is a constant while the second term due to the positive spreading coefficient $\tilde{S}$ decreases linearly with $w_{B}$ as the liquid band widens. This expression simply indicates a spontaneous growth of wetting film without a barrier (i.e., a maximum of $E$ ). This is of course not the case because in the early stage of nucleation (states $\mathrm{i}$ and ii), the liquid band is too narrow for the line and

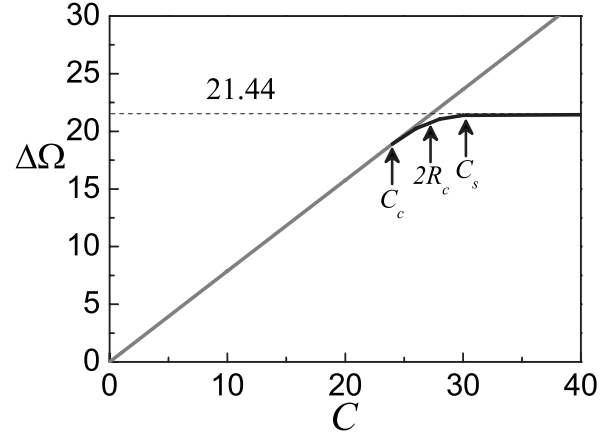

FIG. 4. Grand potential barriers $\Delta \Omega_{D}$ (black) and $\Delta \Omega_{B}$ (gray), each plotted as a function of the circumference of cylindrical substrate $C$, for $\mu_{d}=-0.04$ and $\gamma=0.65$.

surface tensions to be defined. In fact, the barrier is already crossed before the line and surface tensions become valid for sufficiently wide liquid bands.

To further investigate the finite size effect on critical nuclei, we have performed a series of calculations for strip substrates of different widths, i.e., cylindrical substrates of different circumferences, with $\mu_{d}$ and $\gamma$ both fixed. It is physically expected that as the width $C$ is gradually reduced, it will reach a critical value $C_{c}$, below which the DGM of wetting film no longer exists. This nonexistence is numerically verified as follows. By evolving a string toward some stationary MEP, any fluid density variation along the transversal $y$ direction would be gradually removed if $C<C_{c}$. That is, for $C$ below $C_{c}$, a MEP corresponding to the BGM is always obtained in the end. Above $C_{c}$, however, the nucleation may occur through either the BGM or the DGM, with the dominant mode determined by the saddle point of lower $\Omega$. The critical width (circumference) is numerically found to be $C_{c} \cong 24$, a bit smaller than $2 R_{c} \cong 27$, an estimate of $C_{c}$ in the macroscopic description. Shown in Fig. 4 are the grand potential barriers associated with the DGM (black) and BGM (gray), each plotted as a function of $C$. It is readily seen that below $C_{c}$, the nucleation can only occur through the BGM. Above $C_{c}$, however, both the DGM and BGM are available, with $\Delta \Omega_{D}<\Delta \Omega_{B}$. Since the density variation is one dimensional (along $x$ ) in the BGM, $\Delta \Omega_{B}$ shows a linear dependence on $C$, i.e., $\Delta \Omega_{B} \propto C$. For the DGM, it is expected and observed that as $C$ approaches $C_{s} \cong 30, \Delta \Omega_{D}$ saturates at 21.44, which is the value obtained in the case of infinite planar substrate. We want to emphasize that only by using the diffuse-interface model can we obtain the transition from DGM to BGM as $C$ crosses $C_{c}$ from above.

\section{Nucleation on spherical substrates}

Now we turn to the spherical substrates for which the spherical coordinate system is used, with the origin taken at the center of the sphere and $(r, \theta, \varphi)$ denoting the radial, zenith angle, and azimuth angle coordinates, respectively. According to the qualitative analysis in Sec. II B, there exist three distinct kinds of transition pathways of finite barriers, characterized by the layerlike, disklike, and bandlike critical nuclei, respectively. All these critical nuclei possess the rotational symmetry with respect to the azimuth angle $\varphi$, and 

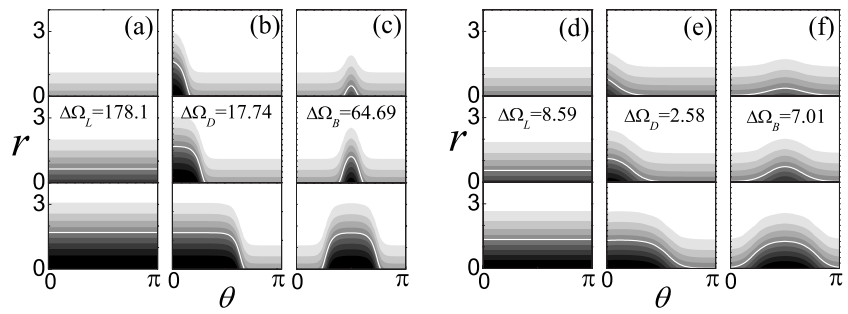

FIG. 5. Representative states picked along transition pathways, with (a)-(c) for $R_{\mathrm{sph}}=20$ and (d)-(f) for $R_{\mathrm{sph}}=10$. In each group, the LGM is on the left, the DGM is at the middle, and the BGM is on the right. Here the relative density $\phi(r, \theta)$ is plotted with $r$ measured from the spherical substrate. The gray scale varies from black for liquid phase $\left(\phi_{+}=1\right)$ to white for vapor phase $\left(\phi_{-}=-1\right)$. In particular, the level curve $\phi=0$ is marked by the white line. For $R_{\mathrm{sph}}=20$ and $\mu_{d}=-0.04, \gamma=0.76$ is used (between $\gamma_{B}=0.75$ and $\left.\gamma_{S}=0.83\right)$. The barriers are $\Delta \Omega_{L}=178.1$ for the LGM, $\Delta \Omega_{D}=17.74$ for the DGM, and $\Delta \Omega_{B}=64.69$ for the BGM. For $R_{\mathrm{sph}}=10$ and $\mu_{d}=-0.04$, $\gamma=0.89$ is used (between $\gamma_{B}=0.87$ and $\gamma_{S}=0.92$ ). The barriers are $\Delta \Omega_{L}=8.59$ for the LGM, $\Delta \Omega_{D}=2.58$ for the DGM, and $\Delta \Omega_{B}=7.01$ for the BGM. It is noted that the liquid-vapor interface appears to be more diffused on the smaller sphere, as seen in (e) and (f).

hence in numerical calculations the relative density $\phi$ is treated as $\phi(r, \theta)$ in a 2D computational domain (with the curvature effects automatically taken into account). With $\mu_{d}=-0.04$ fixed, we first evaluate the binodal $\gamma_{B}$ (at which $\Omega_{V}=\Omega_{F}$ ) and the spinodal $\gamma_{S}$ (at which the vapor phase loses its metastability) for different values of $R_{\mathrm{sph}}$, the radius of the spherical substrate. Compared to the binodal 0.615 and the spinodal 0.76 obtained for planar substrates, ${ }^{28}$ we find that both the binodal $\gamma_{B}$ and the spinodal $\gamma_{S}$ increase with the decreasing radius $R_{\mathrm{sph}}$, and the metastability range of the vapor phase, i.e., $\gamma_{S}-\gamma_{B}$, narrows with the decreasing $R_{\mathrm{sph}}$. Physically, the increase in $\gamma_{B}$ is due to the spherical geometry. That is, for a wetting film covering a sphere, the area of the liquid-vapor interface (contributing a positive free energy) is larger than that of the liquid-solid interface (contributing a negative free energy). Therefore, a smaller $R_{\text {sph }}$ demands a larger $\gamma_{B}$ to ensure $\Omega_{V}=\Omega_{F}$. However an increased $\gamma$ tends to weaken the metastability of the vapor phase [in which the low density at the solid surface is not favored by $\left.\Phi\left(\phi_{s}\right)=-\gamma \phi_{s}\right]$, and hence the metastability range narrows as $R_{\text {sph }}$ decreases. We also find that as long as the vapor phase is metastable (for $\gamma$ between $\gamma_{B}$ and $\gamma_{S}$ ), the three growth modes (namely, the LGM, DGM, and BGM) all exist, characterized by their respective critical nuclei of different barrier heights. Starting from an initial string deliberately prepared for a particular growth mode, we can numerically obtain the MEP corresponding to that mode from the fictitious string evolution in the configuration space. Below we present the MEPs, i.e., the most probable transition pathways, characterized by critical nuclei of distinct types.

We have computed the MEPs for $R_{\mathrm{sph}}=20$ and 10, respectively. A sequence of three states can be found in Fig. 5 for each of the three growth modes. It is seen that for the LGM shown in Figs. 5(a) and 5(d), the wetting film grows up layer by layer along the radial direction, and the critical nucleus is a spherically symmetric liquid film covering the whole substrate. As for the DGM shown in Figs. 5(b) and $5(\mathrm{e})$, the growth of the wetting film starts around $\theta=0$ (a point conveniently chosen for calculation) and continues along the increasing direction of the zenith angle, and the critical nucleus is a curved liquid disk like a cap. Using the excess free energy expressed as $E=l \tilde{\tau}-A \widetilde{S}$ in terms of the line tension $\tilde{\tau}$ and the positive spreading coefficient $\tilde{S}$, we may qualitatively understand this growth mode through the macroscopic description. For a disk bounded at a zenith angle $\theta$, its surface area $A$ and circumference $l$ of it are $A=2 \pi R_{\mathrm{sph}}^{2}(1-\cos \theta)$ and $l=2 \pi R_{\mathrm{sph}} \sin \theta$, respectively. Substituting $A$ and $l$ into $E$ and maximizing $E$ with respect to $\theta$, we obtain the critical zenith angle $\theta_{c}=\cot ^{-1}\left(R_{\mathrm{sph}} \tilde{S} / \widetilde{\tau}\right)$, which is always smaller than $\pi / 2$. It follows that in the macroscopic description, the DGM always exists regardless of the value of $R_{\mathrm{sph}}$, a feature not found for the cylindrical substrates. Moreover, the finite size of the disklike critical nuclei (with $\theta_{c}<\pi / 2$ ) means that even if the binodal $\gamma_{B}$ is approached, the barrier height $\Delta \Omega_{D}$ remains finite. This is distinct from what happens to the planar substrates on which the radius of a disklike critical nucleus $R_{c}=\tilde{\tau} / \tilde{S}$ diverges as the binodal is approached $(\widetilde{S} \rightarrow 0)$, and so does the barrier $E_{c}=\pi \tilde{\tau} / \tilde{S}$.

As for the BGM shown in Figs. 5(c) and 5(f), the growth of the wetting film starts from $\theta=\pi / 2$ (an orthodrome conveniently chosen for calculation) and continues bilaterally along both the increasing and decreasing directions of the zenith angle. The critical nucleus is a narrow liquid band that is symmetric with respect to the orthodrome $\theta=\pi / 2$. There is no macroscopic description for the BGM based on an excess free energy expressed in terms of $\tilde{\tau}$ and $\tilde{S}$. A direct and simple explanation is as follows. A liquid band may span an orthodrome of the sphere (e.g., $\theta=\pi / 2$ ) or not. If it spans the whole orthodrome, then a macroscopically wide liquid band will expand spontaneously with increasing area and decreasing boundary, both of which lead to lower excess free energy. If the orthodrome is not completely covered, then the liquid band will be transformed by the line tension into a liquid disk with a circular boundary. It follows that a macroscopically wide liquid band cannot be stationary on a spherical substrate. Therefore, no macroscopic description exists for the BGM which can only be obtained using the diffuseinterface model. This is similar to what we have for the cylindrical substrates. That is, before the barrier is crossed, the line and surface tensions cannot be defined for the very narrow liquid band.

Finally, we note that among the three coexisting growth modes, the DGM always has the lowest barrier, even if the binodal is approached. That is, although a MEP can be located for each of the three growth modes and by definition that MEP is locally more probable than those pathways nearby, the truly most probable transition pathway is always the one that passes through the disklike critical nucleus.

\section{CONCLUSION}

Using the mean-field diffuse-interface model, we have numerically investigated the various critical nuclei for the nucleation of wetting films on curved substrates. We have introduced three distinct kinds of critical nuclei for three possible growth modes of wetting films, namely, the LGM, DGM, and BGM. By employing the string method, we have 
numerically calculated the various MEPs from which we can obtain the saddle points of grand potential functional and the corresponding nucleation barriers. Our results have shown the following.

- On an infinite planar substrate, a wetting film can be formed with finite probability only by crossing a saddle point characterized by a liquid disk. The radius of this critical nucleus $R_{c}$ is the characteristic length scale over which finite size effects of curved substrates become relevant.

- On a cylindrical substrate of circumference $C$, the disklike critical nucleus exists only if $C$ is above a critical value that is very close to $2 R_{c}$. Below this critical value, the nucleation of wetting film can only occur by crossing a saddle point characterized by a liquid band. This transition from DGM to BGM for $C$ crossing the critical circumference from above is beyond the macroscopic description based on line and surface tensions.

- On a spherical substrate, the three growth modes coexist as long as the vapor phase maintains its metastability. Each growth mode is characterized by a particular critical nucleus, but the DGM always has the lowest nucleation barrier which makes it the truly most probable transition pathway. In particular, the DGM always exists regardless of how small the sphere is, a feature not found for the cylindrical substrates. Moreover, the finite size of the disklike critical nuclei means that the barrier height remains finite even if the binodal is approached, a feature distinct from what happens to the planar substrates.

\section{ACKNOWLEDGMENTS}

This work was supported by Award No. SA-C0040/UKC0016, made by King Abdullah University of Science and Technology (KAUST) and Hong Kong RGC under Grant No. HKUST602007.

${ }^{1}$ J. W. Cahn, J. Chem. Phys. 66, 3667 (1977).

${ }^{2}$ H. Nakanishi and M. E. Fisher, Phys. Rev. Lett. 49, 1565 (1982).

${ }^{3}$ D. Bonn and D. Ross, Rep. Prog. Phys. 64, 1085 (2001).

${ }^{4}$ T. F. Meister and D. M. Kroll, Phys. Rev. A 31, 4055 (1985).

${ }^{5}$ S. Dietrich and M. Schick, Phys. Rev. B 33, 4952 (1986).

${ }^{6}$ S. Dhawan, M. E. Reimel, L. E. Scriven, and H. T. Davis, J. Chem. Phys. 94, 4479 (1991).

${ }^{7}$ A. Patrykiejew and S. Sokołowski, J. Phys. Chem. B 103, 4466 (1999).

${ }^{8}$ R. Bausch and R. Blossey, Europhys. Lett. 14, 125 (1991).

${ }^{9}$ R. Bausch and R. Blossey, Phys. Rev. E 48, 1131 (1993).

${ }^{10}$ R. Bausch, R. Blossey, and M. A. Burschka, J. Phys. A 27, 1405 (1994).

${ }^{11}$ R. Bausch and R. Blossey, Phys. Rev. E 50, R1759 (1994).

${ }^{12}$ E. M. Blokhuis, Phys. Rev. E 51, 4642 (1995).

${ }^{13}$ I. Schmidt and K. Binder, Z. Phys. B: Condens. Matter 67, 369 (1987).

${ }^{14}$ B. M. Law, Phys. Rev. Lett. 69, 1781 (1992).

${ }^{15}$ D. Bonn, H. Kellay, and G. H. Wegdam, Phys. Rev. Lett. 69, 1975 (1992).

${ }^{16}$ D. Bonn and J. O. Indekeu, Phys. Rev. Lett. 74, 3844 (1995).

${ }^{17}$ D. Bonn, E. Bertrand, J. Meunier, and R. Blossey, Phys. Rev. Lett. 84, 4661 (2000).

${ }^{18}$ R. Hołyst and A. Poniewierski, Phys. Rev. B 36, 5628 (1987).

${ }^{19}$ M. P. Gelfand and R. Lipowsky, Phys. Rev. B 36, 8725 (1987).

${ }^{20}$ P. Upton, J. Indekeu, and J. Yeomans, Phys. Rev. B 40, 666 (1989).

${ }^{21}$ T. Gil and L. V. Mikheev, Phys. Rev. E 52, 772 (1995).

${ }^{22}$ M. C. Stewart and R. Evans, Phys. Rev. E 71, 011602 (2005).

${ }^{23}$ A. O. Parry, C. Rascón, and L. Morgan, J. Chem. Phys. 124, 151101 (2006).

${ }^{24}$ W. E, W. Ren, and E. Vanden-Eijnden, Phys. Rev. B 66, 052301 (2002).

${ }^{25}$ W. E, W. Ren, and E. Vanden-Eijnden, J. Chem. Phys. 126, 164103 (2007).

${ }^{26}$ T. Qian, W. Ren, and P. Sheng, Phys. Rev. B 72, 014512 (2005).

${ }^{27}$ C. Qiu and T. Qian, Phys. Rev. B 77, 174517 (2008); 79, 054513 (2009).

${ }^{28}$ C. Qiu, T. Qian, and W. Ren, J. Chem. Phys. 129, 154711 (2008). 